\title{
CÓDIGOS CONVOLUCIONAIS PUNCIONADOS REFINADOS
}

\author{
Bartolomeu F. Uchôa-Filho, Richard Demo Souza, Cecilio Pimentel, Andrei P. Legg, Marcel Jar
}

\begin{abstract}
In this paper, a new class of punctured convolutional codes (PCCs) is presented, in which a PCC is obtained by puncturing a rate $1 / 2$ periodically time-varying convolutional code (the "mother" code), where different phases within a period may have different memory sizes. In the new class, which encompasses all previous PCCs of rate greater than $1 / 2$ ever published, the trellis complexity can be gradually increased resulting in a better distance spectrum at each new complexity increment, offering more flexibility on the choice of the complexity $\times$ performance relationship.
\end{abstract}

Keywords: Punctured convolutional codes, time-varying codes, decoding complexity, distance spectrum.

Resumo - Neste artigo, uma nova classe de códigos convolucionais puncionados (CCPs) é apresentada, na qual um CCP é obtido a partir do puncionamento de um código convolucional (o código "mãe") periodicamente variante no tempo de taxa $1 / 2$, sendo que, dentro de um período, fases diferentes podem ter comprimentos de memória distintos. $\mathrm{Na}$ nova classe, que compreende todos os CCPs de taxa maior do que $1 / 2$ já publicados, a complexidade da treliça pode ser gradualmente incrementada, obtendo-se um melhor espectro de distâncias a cada novo incremento de complexidade, oferecendo uma maior flexibilidade na escolha da relação complexidade $\times$ desempenho.

Palavras-chave: Códigos convolucionais puncionados, códigos variantes no tempo, complexidade de decodificação, espectro de distâncias.

\section{INTRODUÇÃO}

\section{A. Complexidade e Espectro de Distâncias}

Um código convolucional pode ser representado por uma treliça semi-infinita que consiste, após um breve transiente, em cópias concatenadas de uma estrutura básica chamada módulo. McEliece e Lin [1] estabeleceram que o esforço

Bartolomeu F. Uchôa-Filho e Andrei P. Legg são do Grupo de Pesquisa em Comunicações - GPqCom, Departamento de Engenharia Elétrica. Universidade Federal de Santa Catarina, Florianópolis - SC, 88040-900, e-mails: uchoa@eel.ufsc.br, trih@bol.com.br.

Richard Demo Souza é do Programa de Pós-Graduação em Engenharia Elétrica e Informática Industrial - CPGEI. Centro Federal de Educação Tecnológica do Paraná, CEFET/PR. Curitiba - PR, 80230901. e-mail: richard@cpgei.cefetpr.br.

Cecilio Pimentel e Marcel Jar são do Grupo de Pesquisa em Comunicações - CODEC, Departamento de Eletrônica e Sistemas, Universidade Federal de Pernambuco, C.P. 7800 - Recife - PE, 50711-970, e-mails: cecilio@ufpe.br, marceljar@yahoo.com.br.

Este trabalho foi em parte financiado pelo CNPq, através dos processos: 301253/2004-8 e 302286/2004-7. computacional requerido pelo algoritmo de Viterbi para decodificar um bit é proporcional ao número de bits codificados (no módulo) por bit de informação. Chamaremos essa medida de complexidade de treliça, ou CT simplesmente. Um código convolucional de taxa $R=k \cdot / n$ e comprimento de memória $\nu$ (número de registradores de deslocamento do codificador) pode ser representado por um módulo, que chamaremos de módulo convencional, cuja CT cresce exponencialmente com $k+\nu$. Os códigos convolucionais puncionados (CCPs) [2] formam uma classe especial de códigos convolucionais em que a taxa exponencial de crescimento da CT é de apenas $\nu$. Daí o interesse em CCPs, sobretudo nos de altas taxas.

O desempenho de códigos convolucionais com decodificação de máxima verossimilhança, através do algoritmo de Viterbi, pode ser avaliado a partir do espectro de distâncias. Para um código convolucional de taxa $R$, a probabilidade de um evento de erro para o canal com ruído aditivo Gaussiano branco com modulação BPSK, decisão suave e alta relação sinal-ruído é limitada superiormente por[3]:

$$
P_{E} \leq \sum_{d \geq d_{d},} N_{d} Q\left(\sqrt{2 R d \frac{E_{b}}{N_{0}}}\right),
$$

onde $N_{d}$ é o número de sequiências codificadas de peso de Hamming $d, E_{b}$ é a energia do sinal recebido por bit de informação, $N_{0}$ é a densidade espectral de potência unilateral do ruído, e $Q(\cdot)$ é a função definida como $Q(s)=$ $\int_{s}^{\infty} \epsilon^{-t^{2} / 2} d t / \sqrt{2 \pi}$. O espectro de distâncias é definido como a seqüência $\left(N_{d_{f r e}}, N_{d_{t r e t}+1}, \ldots\right)$. Portanto, uma análise de (1) com base em propridades da função $Q(\cdot)$ revela que o melhor espectro é aquele que apresenta o maior valor possível de $d_{\text {free }} \mathrm{e}$, dentre os espectros com o mesmo $d_{\text {free }}$, o melhor é aquele com os menores valores de $N_{d}$ [4]. Em outras palavras, o melhor espectro para uma dada $d_{f, \epsilon e}$ é o primeiro na ordem lexicográfica.

\section{B. Breve Histórico Sobre Códigos Convolucionais Punciona- dos}

Os códigos convolucionais puncionados (CCPs) são uma excelente alternativa aos códigos convolucionais convencionais para o caso de taxas estritamente maiores do que $1 / 2 \mathrm{e}$ quando o algoritmo de Viterbi é adotado para a decodificação de máxima verossimilhança. Cain et al. [2] foram os primeiros a propor uma técnica que consistia na remoção (puncionamento) de alguns dos bits codificados de um código convolucional de baixa taxa (denominado código "mãe") com o objetivo de gerar um código de taxa mais elevada; a escolha do código "mãe" e dos bits a serem removidos era tal que a distância livre $\left(d_{\text {free }}\right)$ do código resultante fosse a maior possível. A motivação para se fazer isso foi justamente a possibilidade de se utilizar a treliça do código "mãe" (desprezando-se as posições que haviam sido removidas) que. 
menos complexa, simplificaria a decodificação via o algoritmo de Viterbi. Em [2], alguns CCPs com melhores espectros foram listados para taxas $2 / 3$ e $3 / 4$, obtidos a partir de um código "mãe" de taxa 1/2.

Yasuda et al. [5] estenderam a idéia de códigos convolucionais puncionados para o caso de altas taxas, em particular taxas na faixa de $2 / 3$ a $13 / 14$. Entretanto, para cada comprimento de memória $\nu, 2 \leq \nu \leq 7$, foi proposto um único código "mãe" otimizado com diferentes puncionamentos, gerando CCPs de diferentes taxas. Esses ficaram conhecidos como códigos rate-compatible [6], e portam a vantagem da adaptabilidade de taxas visto que tendo fixado o código "mãe", exatamente a mesma treliça pode ser adotada para decodificar códigos de diferentes taxas, mudandose apenas a quantidade e as posições dos bits a serem desprezados (bits puncionados).

Numa outra direção, Hole [7] também considerou CCPs de altas taxas porém sem a restrição que fixa o código "mãe", adotada por Yasuda et al. [5]. Como consequêencia desse maior grau de liberdade novos CCPs de altas taxas com melhores $d_{f, \epsilon \in}$ e $N_{d_{f, \ldots}}$ foram tabelados em [7]. Para tais CCPs, bem como para os CCPs publicados em todas as referências supracitadas, apenas códigos "mãe" invariantes no tempo foram considerados. Por outro lado, é sabido que os códigos convolucionais variantes no tempo formam uma classe de códigos mais numerosa. Por exemplo, em [8] e [9], códigos convolucionais periodicamente variantes no tempo melhores (em termos de maior $d_{f r e \epsilon}$ ou de melhor espectro) que os melhores códigos convolucionais invariantes no tempo, com a mesma taxa e a mesma complexidade, foram encontrados. O presente artigo considera CCPs obtidos a partir de códigos "mãe" variantes no tempo. A evolução histórica de tais CCPs será oportunamente descrita ao longo das próximas seções.

\section{Objctivos e Organização do Artigo}

Neste artigo uma nova classe de CCPs, que compreende todos os CCPs de taxa maior do que $1 / 2$ já publicados, é apresentada. Nesta classe, um CCP é obtido a partir do puncionamento de um código convolucional periodicamente variante no tempo de taxa $1 / 2$, sendo que, dentro de um período, fases diferentes podem ter comprimentos de memória distintos. Tal característica possibilita novos CCPs nesta nova classe que podem oferecer uma solução de compromisso mais refinada entre complexidade de decodificação e desempenho. Uma busca computacional é realizada impondo-se um molde à matriz geradora (formalmente definida na Equação (4) da Seção 3). Esse molde fixa 0's e 1's em algumas posições da matriz geradora para garantir uma certa complexidade de decodificação (expressa pela CT), enquanto as demais posições variam livremente durante a busca, que identifica a melhor combinação (melhor espectro de distâncias). Tabelas serão apresentadas contendo novos códigos com uma gama variada de taxas, CTs e espectros de distâncias, preenchendo assim lacunas deixadas em algumas tabelas de CCPs encontradas na literatura.

Este artigo é organizado da seguinte maneira. Na Seção 2 introduzimos os conceitos de módulo de treliça e sua complexidade associada. Códigos convolucionais periodicamente variantes no tempo são revistos na Seção 3 , enquanto que os

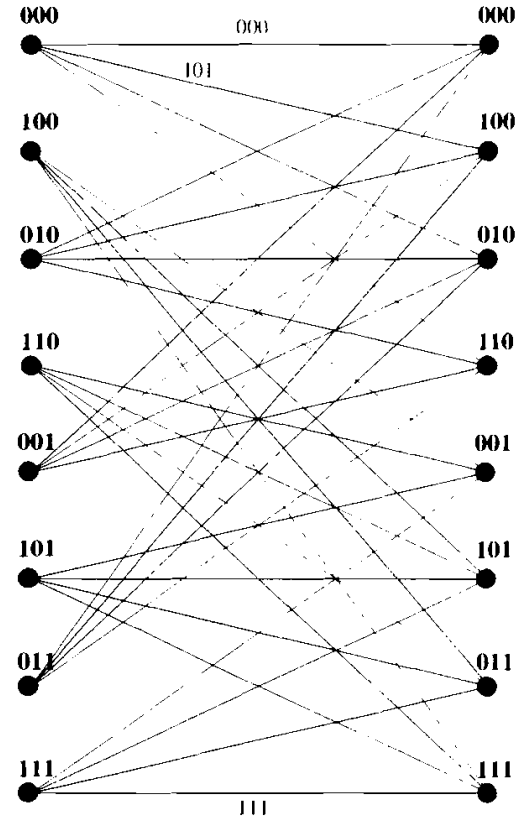

Figura 1. Módulo convencional com $C T\left(M_{\text {conv }}\right)=48$ bits codificados por bits de informação para um código convolucional $(3,2,3)$ que pode ser obtido a partir do puncionamento de um código invariante no tempo de taxa 1/2.

códigos convolucionais puncionados refinados são introduzidos na Seção 4. Nas Seções 5 e 6 discutimos alguns aspectos relacionados ao cálculo do espectro de um código convolucional e da busca computacional efetuada neste trabalho. A Seção 7 apresenta os resultados desta busca computacional, contendo cinco novas tabelas de códigos convolucionais puncionados refinados. Por fim, a Seção 8 conclui o artigo.

\section{MÓDULOS DE TRELIÇA E SUAS COMPLEXIDADES DE TRELIÇA}

\section{A. O Módulo Convencional}

A maneira mais direta de se representar um código convolucional é através do seu módulo convencional, que chamaremos abreviadamente por $M_{\text {conv }}$. Seja $\mathcal{C}$ um código convolucional sobre GF(2), com comprimento de bloco $n$, $k$ bits de informação, comprimento de memória $\nu$ (onde $\nu$ é o menor inteiro tal que $\mathcal{C}$ possa ser obtido por um codificador que requer apenas $\nu$ unidades de atraso) e distância livre $d_{\text {free }} . \mathcal{C}$ é então dito ser um código convolucional $\left(n, k, \nu . d_{\text {free }}\right)$. Quando desnecessários para a discussão, alguns desses quatro parâmetros poderão ser descartados. $O$ módulo convencional para $\mathcal{C}$ consiste em $2^{\nu}$ estados iniciais e $2^{\nu}$ estados finais. Cada estado inicial é conectado por $2^{k}$ ramos direcionados aos estados finais, e cada ramo é rotulado com $n$ bits codificados. O módulo $M_{\text {conv }}$ para um código convolucional $(3.2,3)$ é mostrado na Figura 1. A CT de $M_{\text {conv }}$ é dada por [1]:

$$
C T\left(M_{\text {conv }}\right)=\frac{n}{k} \cdot 2^{\nu+k}
$$




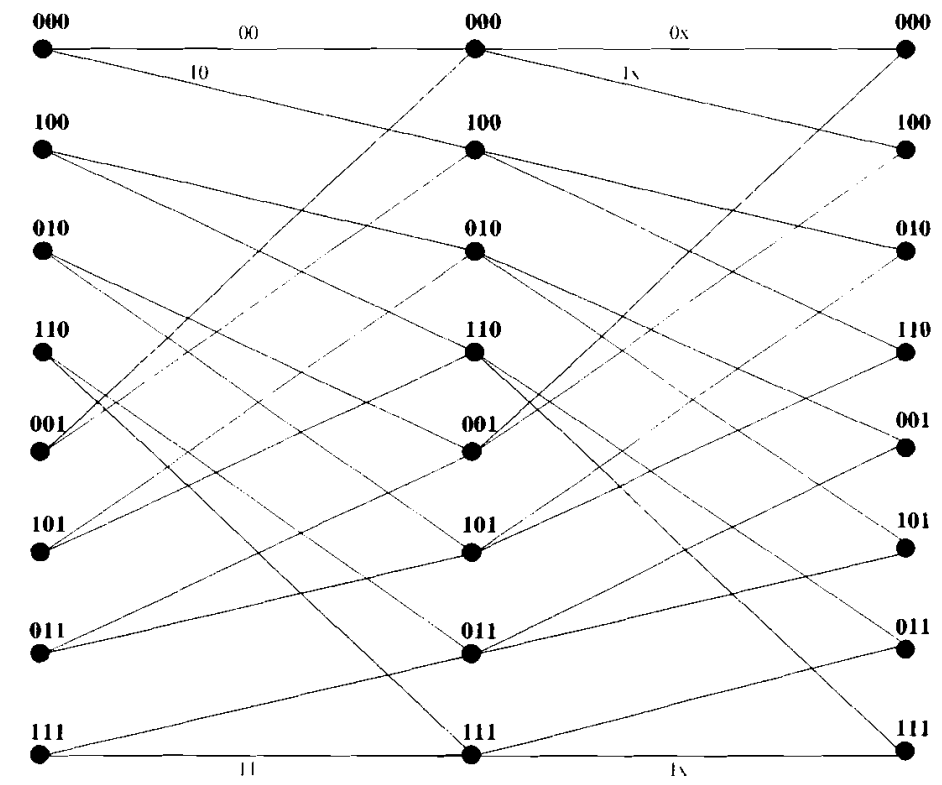

Figura 2. Módulo puncionado com $C T\left(M_{C C P}\right)=24$ bits codificados por bits de informação para o mesmo código $(3,2,3)$ da Figura 1.

bits codificados por bit de informação, medida que cresce exponencialmente com a taxa do código.

\section{B. O Módulo Mínimo}

Todo código convolucional também pode ser representado pelo módulo mínimo, que é obtido a partir da treliça mínima (ou treliça BCJR [10]) de um código de bloco associado ao código convolucional, de acordo com um procedimento apresentado por McEliece e Lin [1]. Para qualquer código convolucional, o módulo mínimo é único, a menos de um isomorfismo, e é também aquele com a menor CT. Por outro lado, o módulo mínimo apresenta uma estrutura topológica bastante irregular, e que nem sempre pode ser pré-determinada a partir de um dado molde imposto à matriz geradora, como é feito neste artigo. Dito de outra maneira, se um único elemento dessa matriz for mudado, porém mantendo-se o mesmo molde imposto à matriz, ou se os bits de saída do codificador convolucional forem permutados, então o módulo mínimo para o código convolucional resultante possivelmente terá uma topologia bastante distinta daquela do módulo mínimo para o código original. A CT da treliça mínima para um código convolucional pode ser obtida a partir da matriz geradora que gera o código, depois de esta ter sido reduzida a uma forma particular, conhecida como minimal spam form [1]. Em geral, não há uma expressão para a CT da treliça mínima que dependa exclusivamente de um dado molde imposto à matriz geradora.

\section{O Módulo Puncionado}

Uma maneira de amenizar a complexidade em (2) para códigos com taxa $k / n>1 / 2$, sem ter que lidar com as dificuldades associadas ao módulo mínimo, mencionadas na subseção anterior, é puncionar um código convolucional de baixa taxa, ou seja um código convolucional $\left(n^{\prime}, 1, \nu\right)$, chamado de código "mãe", e adotar o seu módulo como estrutura básica, a partir da qual se possa construir um módulo de baixa complexidade para o código de alta taxa, que será periódico de período $k$. Esse módulo, que será chamado de módulo puncionado, será denotado por $M_{C C P}$. Podese mostrar que o mesmo código representado pelo módulo $M_{\text {conv }}$ da Figura 1 pode ser obtido a partir do puncionamento de um código invariante no tempo de taxa 1/2. O módulo puncionado $M_{C C P}$ para esse código tem período 2 (fases) e é mostrado na Figura 2, onde " $X$ " denota um bit puncionado. Note nesse módulo que, se não fosse pelo puncionamento na segunda fase, as duas fases seriam idênticas, e assim o código seria invariante no tempo. Na Figura 2, a primeira seção do $M I_{C C P}$ - a seção não puncionada - corresponde exatamente ao módulo do código "mãe". A grande maioria dos CCPs conhecidos foi produzida a partir de códigos "mãe" invariantes no tempo[2, 5,7]. CCPs obtidos a partir de códigos "mãe" variantes no tempo foram considerados pelos autores $[11,12,13]$ e por Bocharova e Kudryashov [14]. O resultado são CCPs com um melhor espectro de distâncias [12, 14] ou com uma menor complexidade de treliça $[11,13]$. A CT de um $\operatorname{CCP}(n, k, \nu)$ qualquer é dada por [1]:

$$
C T\left(M_{C C P}\right)=\frac{n}{k} \cdot 2^{\prime+1}
$$

bits codificados por bit de informação, a qual, para $k>1$, é sempre menor do que (2). Assim, temos que nas Figuras 1 e 2 as complexidades de treliça são, para o mesmo código, $C T\left(M_{\text {conv }}\right)=48$ e $C T\left(M_{C C P}\right)=24$ bits codificados por bits de informação, respectivamente. Na próxima seção, códigos convolucionais periodicamente variantes no tempo serão revistos, porém numa forma mais geral do que aquela apresentada em $[9,8]$. A característica variante no tempo encontrada nos códigos "mãe" apresentados em $[11,12,13,14]$ será refinada, permitindo que fases diferentes possam ter comprimentos de memórias diferentes. As consequiências desse refinamento serão percebidas na Seção 7 . 


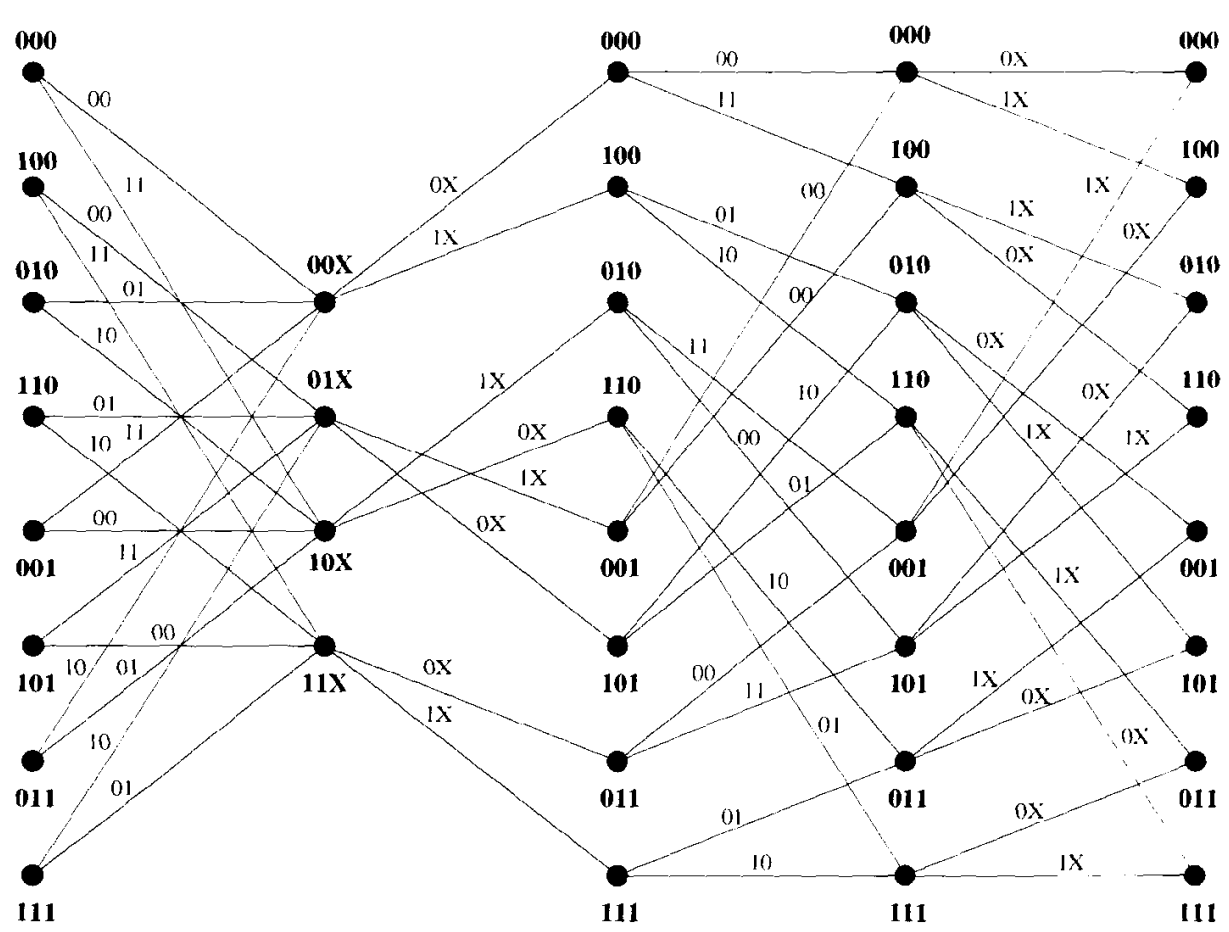

Figura 3. Módulo $M_{C C P R}$ com $C T\left(M_{C C P R}\right)=22$ bits codificados por bits de informação para o código convolucional puncionado refinado do Exemplo 1.

\section{CÓDIGOS CONVOLUCIONAIS PE- RIODICAMENTE VARIANTES NO TEMPO}

Sejam $\mathbf{u}_{t}$ uma $k^{\prime}$-upla binária e $\mathbf{v}_{t}$ uma $n^{\prime}$-upla binária que representam, respectivamente, o sub-bloco de dígitos de informação e o sub-bloco codificado no instante $t$, onde $t \in \mathbb{Z}^{+}$. Por questão de conveniência, $\mathbf{u}_{\left[t_{s} . t_{e}\right)}$ e $\mathbf{v}_{\left[t_{s} . t_{e}\right)}$ denotam, respectivamente, as sequiências de entrada e saída no intervalo de tempo $t_{s}, t_{s}+1, \cdots, t_{e}-2, t_{e}-1$. Assim, a equação de codificação de um código convolucional periodicamente variante no tempo (CCPVT) $\left(n^{\prime}, k^{\prime}\right)$ de período $P$ (fases) é dada por $\mathbf{v}_{[0, \infty)}=\mathbf{u}_{[0, \infty)} G_{\text {escalar }}$, onde

$$
G_{\text {escalar }}=\left[\begin{array}{ccccccc}
\ddots & & & & & & \\
& G_{\nu_{0}}^{0} & & & & & \\
\ddots & \vdots & \ddots & & & & \\
\ddots & G_{1}^{0} & & G_{\nu_{p}}^{p} & & & \\
& G_{0}^{0} & \ddots & \vdots & \ddots & & \\
& & \ddots & G_{1}^{p} & & G_{\nu_{P-1}}^{P-1} & \\
& & & G_{0}^{p} & \ddots & \vdots & \ddots \\
& & & & \ddots & G_{1}^{P-1} & \\
& & & & & G_{0}^{P-1} & \ddots \\
& & & & & & \ddots
\end{array}\right]
$$

é a matriz geradora semi-infinita ou matriz geradora escalar do CCPVT. Em (4), $G_{0}^{[t]_{P}}, G_{1}^{[t]_{P}}, \cdots, G_{\nu_{[t]_{P}}}^{[t]_{P}}$ são matrizes binárias $k^{\prime} \times n^{\prime}$. chamadas de sub-matrizes geradoras no instante $t$, onde $[t]_{P}$ denota $t(\bmod P)$ e é também um índice de fase, e $\nu_{p}$ é o comprimento de memória da fase $p$, que neste trabalho depende de $p$. Quando $P=1$, tem-se um código convolucional inariante no tempo.

\section{CÓDIGOS CONVOLUCIONAIS PUN- CIONADOS REFINADOS}

A seguir, uma forma mais geral de um CCP - o CCP refinado (CCPR) - será descrita. Casos particulares serão oportunamente apresentados. Considere um CCPVT de período $P=l k$ e de taxa $k^{\prime} / n^{\prime}=1 / 2$, onde $l$ é um inteiro positivo. Para se conseguir a taxa desejada, igual a $k / n>1 / 2$, devemos ter exatamente $l(2 k-n)$ fases puncionadas em um bit e $l(n-k)$ fases não puncionadas.

Sejam $I_{P} \subset \mathbb{Z}_{P} \stackrel{\text { def }}{=}\{0,1,2, \ldots, P-1\}$ e $I_{N}=\mathbb{Z}_{P} \backslash I_{P}$ os conjuntos de índices das fases puncionadas e não puncionadas, respectivamente, onde $\left|I_{P}\right|=l(2 k-n)$ e $\left|I_{N}\right|=$ $l(n-k)$. No módulo para esse CCP, na fase puncionada $p$, $p \in I_{P}$, existem $2^{\nu_{p}+1}$ símbolos, e na fase não puncionada $p$, $p \in I_{N}$, existem $2^{\nu_{p}+2}$ símbolos. Portanto, a CT do módulo $M_{C C P R}$, o módulo para o CCPR de taxa $k / n$ obtido a partir do puncionamento de um CCPVT de período $P=l \mathrm{k}$ e de taxa $k^{\prime} / n^{\prime}=1 / 2$, segundo o procedimento acima, é dada por:

$$
C T\left(M_{C C P R}\right)=\frac{1}{l k}\left[\sum_{p \in I_{P}} 2^{\nu_{p}+1}+\sum_{p \in I_{N}} 2^{\nu_{p}+2}\right]
$$

bits codificados por bits de informação. Considere um CCP convencional de taxa $k / n$ com comprimento de memória $\nu$ 
cujo módulo $M_{C C P}$ tem CT dada em (3). Obviamente o módulo $M_{C C P P_{R}}$ será menos complexo do que $M_{C C P}$ se $l^{\prime} p<l$ para pelo menos uma fase $p \in \mathbb{Z}_{P}$, enquanto as outras fases têm comprimento de memória igual a $\nu$. Por outro lado, se $\nu_{p}>\nu$ para pelo menos uma fase então existirá mais espaço para se encontrar códigos com melhor espectro de distâncias.

Example 1 Considere $\quad$ In! $C C P R$ com os seguintes parâmetros: $R=2 / 3 . P=4 . l=2, \nu_{0}=\nu_{2}=\nu_{3}=3$. $\nu_{1}=2$, e sub-matrizes geradoras: fase $0: G_{0}^{0}=[11]$ $G_{r_{1}^{0}}^{(0)}=[00], G_{2}^{0}=[01], G_{3}^{0}=[11] ;$ fase $1: G_{0}^{1}=[1 X]$. $G_{1}^{1}=\left[1 X^{-}\right], G_{2}^{1}=[1 X] ;$ fase $2: G_{0}^{2}=[11], G_{1}^{2}=[01]$. $G_{2}^{2}=[11], G_{3}^{2}=[11]$ : fase $3: G_{0}^{3}=[1 \mathrm{X}], G_{1}^{3}=[1 \mathrm{X}]$. $G_{2}^{3}=[0 \mathrm{X}], G_{3}^{3}=[1 \mathrm{X}]$. A matriz geradora escalar nesse caso é dada por:

$$
G_{\text {escalar }}=\left[\begin{array}{ccccccc}
\ddots & & & & & \\
& {[11]} & & & & \\
& {[01]} & & & & \\
\ddots & {[00]} & {[1 X]} & {[11]} & & \\
& {[11]} & {\left[1 X^{\prime}\right]} & {[11]} & {[1 X]} & \\
& & {[1 X]} & {[01]} & {[0 X]} & \ddots \\
& & & {[11]} & {[1 X]} & \\
& & & & {[1 X]} & \\
& & & & & \ddots
\end{array}\right]
$$

A complexidade de treliça para este código é $C T\left(M_{C C P R}\right)=22$ bits codificados por bits de informação. O módulo $M_{C C P R}$ que o representa pode ser visto na Figura 3. Em relação à treliça puncionada da Figura 2 a redução de complexidade é de quase 10\%. sendo que em relação à treliça convencional da Figura 1 a redução é de mais de $50 \%$.

\section{O CÁLCULO DO ESPECTRO DE DISTÂNCIA}

Todo CCPVT pode ser equivalentemente descrito como um código invariante no tempo [11]. Isso inclui, é claro, a classe dos CCPRs (e, consequientemente, a classe dos CCPs), pois esses são variantes no tempo. Por exemplo, o CCP associado ao módulo puncionado da Figura 2 pode ser equivalentemente representado pela treliça invariante no tempo obtida a partir do módulo $M_{\text {conv }}$ da Figura 1 . O cálculo do espectro de distâncias dos novos códigos apresentados neste artigo foi baseado na treliça invariante no tempo equivalente para estes códigos. Segundo Tang et al. $[15,16]$, o espectro de distâncias de um CCPVT obtido a partir da treliça invariante no tempo é pior, no sentido de que apresenta multiplicidades maiores, do que o espectro obtido a partir da treliça mínima para o mesmo código. Fato semelhante ocorre com relação ao espectro de distâncias a partir da treliça puncionada. Entretanto, conforme relatado em $[15,16]$, para alguns exemplos de códigos convolucionais, os espectros obtidos a partir das duas treliças diferiram apenas a partir da quinta linha espectral. Um estudo mais amplo para avaliar as discrepâncias 76 entre os dois espectros para um código qualquer ainda está por ser realizado. Sendo assim, ainda é possível que alguns CCPRs melhores do que os CCPs listados em [14] existam mas, devido a essa limitação no procedimento de busca aqui adotado, estes não puderam ser encontrados e, conseqüentemente, não foram listados nas tabelas da Seção 7 . Foram listados apenas os CCPRs que apresentaram alguma vantagem com relação aos CCPs listados em [14], ou em termos de melhor espectro de distância (obtido a partir da treliça invariante no tempo) ou em termos de menor CT. A única exceção, apenas para ressaltar o fato acima, pode ser encontrada na Tabela 2 onde, para alguns casos, mesmo aumentando-se a complexidade de treliça não foi possível melhorar o espectro.

\section{A BUSCA COMPUTACIONAL}

Uma busca computacional exaustiva para se encontrar novos CCPRs é impraticável, mesmo que se mantenha ao longo da busca um certo padrão de puncionamento fixo. Por outro lado, o número de maneiras pelas quais o CCPVT descrito acima pode ser puncionado para se alcançar taxa $k / n$ é exatamente:

$$
\frac{(l k) !}{(l(n-k)) ! \times(l(2 k-n)) !}
$$

Para se obter o melhor código, seria necessário considerar todas essas possibilidades. Entretanto, uma busca computacional restrita a apenas alguns padrões de puncionamento pode conduzir a bons códigos. Restrições adicionais (ver final da seção) podem ser impostas que limitam ainda mais a busca sem impedir que bons resultados apareçam, o que será verificado na próxima seção. Por exemplo, se o objetivo for encontrar um CCPR com complexidade menor do que a de um CCP com comprimento de memória $\nu$, então podemos adotar as seguintes restrições:

- $G_{0}^{p} \neq\left[\begin{array}{ll}0 & 0\end{array}\right]$, onde $0 \leq p<P$, para se obter uma distância livre grande[17];

- $\nu_{p} \leq \nu$, onde $0 \leq p<P$, para se ter uma $C T\left(M_{C C P R}\right)$ menor;

- $\nu_{p}+1 \geq \nu_{p+1}$, onde $0 \leq p<P-1$ e $\nu_{P-1}+1 \geq \nu_{0}$, para se conseguir o casamento topológico entre uma fase e a seguinte no módulo.

\section{RESULTADOS}

Nesta seção são apresentados alguns CCPRs com complexidades diversas. Alguns CCPs apresentados em [14] são também listados, para fins de comparação. Os resultados são mostrados nas Tabelas 1-5. Apenas o caso de $l=1$ foi considerado. Os comprimentos de memória em cada fase, $\left(\nu_{0}, \nu_{1}, \ldots, \nu_{P-1}\right)$, a distância livre, $d_{f, \epsilon \epsilon}$, a CT do módulo, as sub-matrizes geradoras do código na forma octal e o espectro de distâncias (três primeiras linhas espectrais) são mostrados nas tabelas. Na coluna das sub-matrizes geradoras, os números octais entre parênteses representam 
Tabela 1. CCPRs para Taxa $R=3 / 4$.

\begin{tabular}{||c|c|c|c|l||}
\hline \hline$\left(\nu_{0}, \ldots, \overline{\nu_{P-1}}\right)$ & $d_{\text {free }}$ & $C T$ & Sub-Matrizes Geradoras & Espectro \\
\hline \hline$(3,3,3)$ & 4 & 21,3 & $15,17,(17,15)$ & 290532 \\
$(4,3,3)$ & 4 & 32,0 & $(25,36), 17,17$ & 1042194 \\
$(4,4,4)$ & 4 & 42,7 & $31,31,(35,23)^{a}$ & 542134 \\
$(4,4,4)$ & 4 & 42,7 & $(27,32), 37,27^{b}$ & 539151 \\
$(4,4,5)$ & 4 & 53,3 & $(35,26), 25,61^{b}$ & 536152 \\
$(5,4,4)$ & 4 & 64,0 & $(65,46), 27,37^{b}$ & 22296 \\
$(5,5,5)$ & 5 & 85,3 & $65,65,(47,61)^{a}$ & 1371326 \\
\hline \hline
\end{tabular}

Tabela 2. CCPRs para Taxa $R=3 / 5$.

\begin{tabular}{|c|c|c|c|c|}
\hline$\left(\nu_{0}, \ldots, \nu_{P}-1\right)$ & $d_{\text {free }}$ & $C T$ & Sub-Matrizes Geradoras & Espectro \\
\hline$(2,2,2)$ & 4 & 13,3 & $7,(7,5),(7,5)^{a}$ & 31224 \\
\hline$(2,2,3)$ & 4 & 18,6 & $5,(5,7),(13,17)^{b}$ & 31224 \\
\hline$(2,3,3)$ & 4 & 24,0 & $7,(13,15),(13,16)^{b}$ & 11232 \\
\hline$(3,3,3)$ & 4 & 26,7 & $15,(15,13),(15,11)$ & 1514 \\
\hline$(4,3,3)$ & 4 & 32,0 & $23,(15,13),(11,16)$ & 1513 \\
\hline$(3,4,3)$ & 5 & 37,3 & $11,(37,22),(13,15)^{b}$ & 41334 \\
\hline$(3,4,4)$ & 5 & 48,0 & $15,(37,24),(23,35)^{b}$ & 41129 \\
\hline$(4,4,4)$ & 6 & 53,3 & $25,(35,21),(37,27)^{a}$ & 180139 \\
\hline$(5,4,4)$ & 6 & 64,0 & $75,(35,21),(27,23)^{b}$ & 180139 \\
\hline$(5,5,5)$ & 6 & 106,7 & $47,(47,65),(45,57)^{a}$ & 11640 \\
\hline
\end{tabular}

as fases não puncionadas. Para o espectro de distâncias

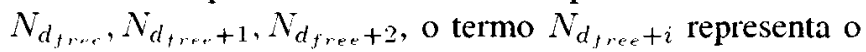
número total de sequêencias codificadas com peso de Hamming igual a $d_{\text {free }}+i$, para $i=0,1,2$. Pode-se perceber a partir das tabelas que é possível obter espectros melhores com pequenos incrementos de complexidade. Esses resultados expandem consideravelmente a classe dos CCPs e oferecem uma seleção mais refinada da relação complexidade $x$ desempenho. Por fim, um resultado incidental. Na Tabela 1, nota-se que um CCP de taxa 3/4 e comprimentos de memória $(4,4,4)$ com espectro melhor do que aquele listado em [14] foi encontrado com a busca realizada neste artigo.

\section{CONCLUSÃO E COMENTÁRIOS FI- NAIS}

Neste artigo um refinamento de códigos convolucionais puncionados, que oferece uma maior flexibilidade na escolha da relação complexidade $\times$ desempenho, foi proposto. Os novos códigos são obtidos a partir do puncionamento de um código convolucional "mãe" periodicamente variante no tempo de taxa $1 / 2$, sendo que em cada período fases diferentes podem ter comprimentos de memória diferentes. Logo, essa classe inclui todos os códigos convolucionais puncionados já publicados como casos particulares. Na nova classe, a complexidade da treliça pode ser gradualmente incrementada, obtendo-se um melhor espectro a cada novo incremento de complexidade.

É importante observar que os novos códigos listados nas tabelas podem apresentar espectros de distâncias ainda melhores, se estes forem calculados a partir da treliça mínima, e não com base na treliça invariante no tempo equivalente, como foi feito neste artigo. Isso se deve a uma propriedade da treliça mínima para códigos convolucionais [15, 16]. Por outro lado, da forma como neste artigo é feita a escolha dos novos códigos, fica subentendida a utilização da treliça refinada para a decodificação de máxima verossimilhança. A complexidade da treliça mínima para um código qualquer é comprovadamente a menor de todas. A relação entre as complexidades da treliça mínima e da treliça refinada para os códigos convolucionais puncionados refinados, bem como a busca de novos códigos com base na complexidade da treliça mínima são problemas de pesquisa interessantes, e que estão sendo investigados pelos autores.

\section{REFERÊNCIAS}

[1] R. J. McEliece and W. Lin, "The trellis complexity of convolutional codes," IEEE Trans. Inform. Theorn, vol. 42, no. 6, pp. 1855-1864, Nov, 1996.

[2] J. B. Cain, G. C. Clark, Jr., and J. M. Geist, "Punctured convolutional codes of rate $(\mathrm{n}-1) / \mathrm{n}$ and simplified maximum likelihood decoding," IEEE Trans. Inform. Theory, vol. IT-25, pp. 97-100, Jan. 1979.

[3] A. J. Viterbi, "Convolutional codes and their performance in communication systems," IEEE Trans. Commun. Technol., vol. COM-19, pp. 751-772, Oct. 1971.

[4] P. Frenger. P. Orten, and T. Ottosson, "Convolutional codes with optimum distance spectrum." IEEE Commun. Lett., vol. 3, pp. 317-319, Nov. 1999.

[5] Y. Yasuda, K. Kashiki, and Y. Hirata, "High-rate punctured convolutional codes for soft-decision Viterbi algorithm." IEEE Trans. Commun., vol. COM-32, no. 3, pp. 315-319, Mar. 1984.

[6] J. Hagenauer, "Rate-compatible punctured convolutional codes and their applications," IEEE Trans. Commun.. vol. 
Tabela 3. CCPRs para Taxa 4/5

\begin{tabular}{|c|c|c|c|l||}
\hline \hline$\left(\nu_{0}, \ldots, \nu_{P-1}\right)$ & $d_{f r e c}$ & $C T$ & Sub-Matrizes Geradoras & Espectro \\
\hline \hline$(2,2,2,2)$ & 2 & 10,0 & $5,5,7,(7,5)$ & 11254 \\
$(2,2,2,3)$ & 2 & 14,0 & $7,7,5,(13,17)$ & 11253 \\
$(2,3,3,3)$ & 2 & 18,0 & $5,13,13,(11,13)^{b}$ & 1947 \\
$(3,3,3,3)$ & 3 & 20,0 & $15,11,11,(11,17)^{a}$ & 632185 \\
$(3,3,3,4)$ & 3 & 28,0 & $17,15,11,(27,32)^{b}$ & 323145 \\
$(3,4,4,4)$ & 3 & 36,0 & $11,37,31,(35,36)^{b}$ & 121139 \\
$(4,4,4,4)$ & 4 & 40,0 & $27,33,27,(37,05)^{a}$ & 30126815 \\
$(4,4,4,5)$ & 4 & 56,0 & $33,27,31,(53,76)^{b}$ & 2092646 \\
$(5,5,5,5)$ & 4 & 80,0 & $75,75,71,(67,41)^{a}$ & 446295 \\
\hline \hline
\end{tabular}

Tabela 4. CCPRs para Taxa $R=4 / 7$.

\begin{tabular}{||c|c|c|c|c||}
\hline \hline$\left(\nu_{0}, \ldots, \nu_{P-1}\right)$ & $d_{\text {free }}$ & $C T$ & Sub-Matrizes Geradoras & Espectro \\
\hline \hline$(3,3,3,3)$ & 5 & 28,0 & $13,(17,11),(17,13),(17,11)$ & 51436 \\
$(3,4,3,3)$ & 5 & 36,0 & $13,(32,25),(17,13),(17,11)$ & 41229 \\
$(3,4,4,3)$ & 5 & 44,0 & $13,(32,25),(37,26),(17,11)^{b}$ & 21036 \\
$(4,4,4,3)$ & 6 & 48,0 & $33,(32,25),(36,27),(15,16)^{b}$ & 280163 \\
$(3,4,4,4)$ & 6 & 52,0 & $11,(32,27),(34,27),(33,27)^{b}$ & 220148 \\
$(4,4,4,4)$ & 6 & 56,0 & $31,(31,25),(35,25),(37,35)^{a}$ & 83052 \\
\hline
\end{tabular}

Tabela 5. CCPRs para Taxa $R=5 / 7$.

\begin{tabular}{||c|c|c|c|l||}
\hline \hline$\left(\nu_{0}, \ldots, \nu_{P-1}\right)$ & $d_{\text {free }}$ & $C T$ & Sub-Matrizes Geradoras & Espectro \\
\hline \hline$(3,3,3,3,3)$ & 4 & 22,4 & $15,(15,11), 13,17,(17,11)$ & 1749205 \\
$(3,3,3,4,4)$ & 4 & 32,0 & $15,(15,11), 13,37,(33,35)$ & 1156166 \\
$(4,4,3,4,4)$ & 4 & 41,6 & $25,(35,23), 13,17,(33,35)^{b}$ & 640148 \\
$(4,4,4,4,4)$ & 4 & 44,8 & $33,(33,21), 31,23,(33,35)^{a}$ & 227109 \\
$(4,4,4,5,5)$ & 4 & 64,8 & $33,(33,23), 31,47,(75,46)^{b}$ & 12183 \\
$(5,5,4,5,5)$ & 5 & 83,2 & $75,(57,76), 27,51,(67,51)^{b}$ & 1667275 \\
$(5,5,5,5,5)$ & 5 & 89,6 & $75,(57,75), 57,55,(67,51)^{a}$ & 1045202 \\
\hline
\end{tabular}

COM-36, pp. 389-400, Apr. 1988.

[7] K. J. Hole. "New short-constraint length rate $(\mathrm{n}-1) / \mathrm{n}$ punctured convolutional codes for soft-decision Viterbi decoding," IEEE Trans. Inform. Theon, vol.IT-34, pp. 1079-1081, Sept. 1988.

[8] R. Palazzo Jr., "A time-varying convolutional encoder better than the best time-invariant encoder," IEEE Trans, on Inform. Theory, vol. 39. no. 3, pp. 1109 - 1110, May 1993.

[9] P. J. Lee,"There are many good periodically time-varying convolutional codes," IEEE Trans, Inform. Theory, vol. 35, no. 2, pp. 460-463, Mar. 1989.

[10] L. R. Bahl, J. Cocke, F. Jelinek, and J. Raviv, "Optimal decoding of linear codes for minimizing symbol error rate," IEEE Trans. Inform. Theory, vol. IT-20, pp. 284-287, Mar. 1974.

[11] B. F. Uchôa-Filho, R. Palazzo. Jr., A. Said, and C. de Almeida, "New unit-memory codes obtained by puncturing periodically time-varying convolutional codes", in SBT/IEEE International Telecommunications Symposium, pp. 534-538, São Paulo, SP. Brazil, Aug. 1998.

[12] B. F. Uchôa-Filho and R. Palazzo, Jr., "Unit-memory codes with simplified maximum likelihood decoding", in ISITA '2000 Intemational Symposium on Information Theor and its Applications, pp. 13-16, Honolulu, HI, U.S.A., Nov. 2000.

[13] R. D. Souza and B. F. Uchôa-Filho, "On trellis modules for convolutional codes," in 2002 IEEE Intemational Symposium on Information Theory, pp. 417, Lausanne, Switzerland, July, 2002.
[14] I. E. Bocharova and B. D. Kudryashov, "Rational rate punctured convolutional codes for soft-decision Viterbi decoding," IEEE Trans. Inform. Theory, vol. 43, no. 4, pp. 1305-1313, July 1997.

[15] H.-H. Tang, M.-C. Lin, and B. F. Uchôa-Filho, "Equivalent convolutional codes with distinct memory sizes of encoders," Anais do ISITA'04, Parma, Itália. Oct. 2004.

[16] H.-H. Tang, M.-C. Lin, and B. F. Uchôa-Filho, "Equivalent convolutional codes with distinct memory sizes of encoders," IEEE Trans. Inform. Theory, aceito para publicação.

[17] R. Johannesson and K. S. Zigangirov. Fundamentals of Convolutional Coding, IEEE Press, New York, NY, 1999.

Bartolomeu Ferreira Uchôa-Filho nasceu em Recife, em 29 de outubro de 1965. Recebeu o título de Engenheiro Eletricista pela Universidade Federal de Pernambuco (UFPE), em 1989; o de Mestre em Engenharia Elétrica pela Universidade Estadual de Campinas (UNICAMP), Campinas, em 1992; e o de Ph.D. em Engenharia Elétrica pela University of Notre Dame, Indiana, U.S.A., em 1996. De 1997 a 1999 realizou Pós-Doutorado na UNICAMP, onde ministrou cursos de Teoria de Informação e Comunicações Digitais. De agosto de 1999 a janeiro de 2000 foi Pesquisador Visitante no Departamento de Engenharia Elétrica da Universidade Federal de Santa Catarina (UFSC), e desde fevereiro de 2000 é Professor Adjunto dessa instituição. Dr. Uchôa foi membro da comissão técnica de várias edições do Simpósio Brasileiro de Telecomunicações, e tem 
atuado como revisor de várias revistas, como IEEE Transactions on Information Theory, IEEE Transactions on Communications, IEEE Transactions on Wireless Communications e a Revista da Sociedade Brasileira de Telecomunicações (RevSBrT). Foi Editor de Circulação e Marketing (2001-2002) e Editor-Chefe (2003-2004) da RevSBrT. É Pesquisador 2 e consultor ad hoc do CNPq, e coordenou dois projetos de pesquisa financiados por aquele conselho. Seus interesses de pesquisá são na área de teoria de informação e codificação, com aplicações em comunicações digitais e sem fio. Dr. Uchôa é membro do Eta Kappa Nu (U.S.A.). da IEEE Information Theory Society, e da Sociedade Brasileira de Telecomunicações.

Richard Demo Souza nasceu em Florianópolis em 09 de março de 1978. Obteve os títulos de Bacharel e Doutor em Engenharia Elétrica pela Universidade Federal de Santa Catarina em 1999 e 2003, respectivamente. Em 2003 foi Pesquisador Visitante do Departamento de Engenharia Elétrica e da Computação da University of Delaware. Newark-DE, EUA. Desde 2004 é Professor Adjunto do Departamento Acadêmico de Eletrônica e do Programa de PósGraduação em Engenharia Elétrica e Informática Industrial, do Centro Federal de Educação Tecnológica do Paraná. Suas áreas de interesse são teoria da codificação, comunicações sem fio e processamento digital de sinais.

Cecilio Pimentel nasceu em Recife em 07 de junho de 1966. Graduou-se em Engenharia Elétrica pela Universidade Federal de Pernambuco em 1987. Recebeu o título de Mestre em Engenharia Elétrica pelo Centro de Estudos em Telecomunicações da Universidade Católica do Rio de Janeiro em 1990 e o título de doutor em Engenharia Elétrica pela University of Waterloo, Canadá, em 1996. Desde 1998 é professor do Departamento de Eletrônica e Sistemas da Universidade Federal de Pernambuco. Seus principais interesses em pesquisa são teoria da informação, dinâmica simbólica, teoria das comunicaçōes. Em 1999. Dr. Pimentel recebeu o prêmio Santista juventude em telecomunicações.

Andrei Piccinini Legg nasceu em Caxias do Sul, em 8 de setembro de 1980. Durante os anos de 2003 e 2004 foi bolsista de iniciação cientifica, com pesquisa na área de códigos. Recebeu o título de Engenheiro Eletricista pela Universidade Federal de Santa Catarina (UFSC), em 2005: Começou o mestrado em março de 2005 na Universidade de Santa Catarina, vinculado ao laboratório Gpqcom, sobre orientação do Dr. Bartolomeu Ferreira Uchôa-Filho. Seus interesses de pesquisa são na área de teoria de informação e codificação, com aplicações em comunicações digitais e sem fio.

Marcel Jar nasceu em Recife em 31 maio de 1982. Graduou-se em Engenharia Eletrônica em março de 2005 pela Universidade Federal de Pernambuco. Atualmente é aluno de mestrado do Programa de Pós-Graduação em Engenharia Elétrica da Universidade Federal de Pernambuco. Seus interesses de pesquisa incluem códigos corretores de erros, processamento de sinais e teoria da informação. 